vasculopathy have limited brain injury (cystic infarction and lacunae), and patients with extensive vasculopathy (stenosis or occlusion) have severe brain injury (encephalomalacia). Brain injury in SCD may be preventable if the anemia and vasculopathy are corrected and reversed.

\title{
POSTTRAUMATIC NONHEMIC SUBDURAL EFFUSION
}

The time course for the development of subdural fluid collections after a single traumatic event in 55 head trauma patients younger than 3 years was determined by CT scan in a retrospective consecutive case series at Children's Hospital of Wisconsin, Milwaukee. The date of injury could be determined for $55(64 \%)$ of 86 patients with trauma-related subdural fluid collections. The mean age for the 55 patients ( 33 boys, 22 girls) was $8.4+/-13.1$ months). The mechanism of injury was intentional trauma in 41 $(75 \%)$, unintentional trauma in $10(18 \%)$, and uncertain intent in $4(7 \%)$. The initial visualization of subdural fluid in 267 CT examinations occurred during the first week after injury in $44(80 \%)$ of 55 patients. In the remaining 11 patients, the time of origin of subdural fluid was less than 2 weeks for 4 and less than 3 weeks for 6 . The location of the fluid was frontal in all but 1 patient $(98 \%)$. The fluid collection was unilateral in $9(16 \%)$ and bilateral in $46(84 \%)$. Hemorrhage accompanying the subdural effusion was subdural in $48(87 \%)$ patients, epidural in $3(6 \%)$, and subarachnoid in $1(2 \%)$. Eight $(15 \%)$ patients died within 30 days of injury. Subdural fluid collections persisted for at least 1 month in 10 $(18 \%)$, spontaneous resolution occurred in $14(25 \%)$, and subdural shunts were placed in 8 $(15 \%)$. Brain atrophy, with enlarged ventricles and sulci, developed in $16(48 \%)$ of 33 patients followed with CT for at least 2 weeks after injury. (Wells RG, Sty JR. Traumatic low attenuation subdural fluid collections in children younger than 3 years. Arch Pediatr Adolesc Med October 2003;157:1005-1010). (Reprints: Robert G Wells MD, Department of Radiology, Children's Hospital of Wisconsin, Mail Stop 721, 9000 W Wisconsin Ave, PO Box 1997, Milwaukee, WI 53201).

COMMENT. Low attenuation subdural fluid on CT in infants with a history of head injury should raise the suspicion of child abuse. The appearance of subdural fluid most often occurs within a few days of the trauma.

Neuroimaging of intraparenchymal lesions predicted outcome in shaken baby syndrome in 23 children with nonaccidental head injury. (Bonnier $\mathrm{C}$ et al. Pediatrics October 2003;112:808-814). A low Glasgow Coma Scale score, retinal hemorrhages, skull fracture, cranial growth deceleration, and brain atrophy on MRI (15 days to 3 months after injury) were significantly associated with poor developmental outcome.

Abusive head injury as a cause of apparent life-threatening events (ALTE) in infancy was diagnosed in $6(2.5 \%)$ of a consecutive series of 243 infants younger than 12 months admitted to Westchester Medical Center, New York Medical College, Valhalla (Altman RL et al. Arch Pediatr Adolesc Med October 2003;157:1011-1015). ALTE is a sudden breathing abnormality, color change, or altered muscle tone or mental status, often requiring emergency resuscitation. Thirty-five different causes of ALTE were identified, and abusive head injury was responsible in 1 admission every 5 months. An ophthalmologic examination revealed retinal hemorrhages leading to a CT scan in 4 of the 
6 diagnosed with abusive head injury and focal seizures developed in 1. ALTE is to be distinguished from sudden infant death syndrome.

A distinct cytokine profile identified in SIDS brain could cause disturbed homeostatic control of cardiorespiratory brainstem centers and arousal responses and might represent a common denominator in this multifactorial syndrome. (Kadhim $\mathrm{H}$ et al. Neurology November 11, 2003;61:1256-1259). The authors detected high neuronal interleukin (IL)-1 [beta] immunoreactivity in the arcuate and dorsal vagal nuclei of SIDS cases.

\section{MOVEMENT DISORDERS}

\section{LONG-TERM OUTCOME OF GILLES DE LA TOURETTE SYNDROME}

Videotapes recorded 1978 through 1991 of 56 children (ages 8 to 14) with Gilles de la Tourette syndrome (GTS) were reviewed and 31 of the patients (28 men and 3 women, age $>20$ years) were recruited for a second videotape and in-person assessment at Rush-Presbyterian-St Luke's Movement Disorder Center, Chicago, IL. Tics were rated for body areas involved, motor and phonic tic frequency, and motor and phonic tic severity. Ninety percent of patients still had tics as adults, and $50 \%$ had objective evidence of tics. Adult patients who considered themselves free of tics were frequently found to exhibit tics on objective assessment. Despite this perceived disparity, the objective tic disability was significantly diminished in adults compared to that in childhood. All tic domains (body area involved, frequency, severity) had improved, and especially motor tic severity $(\mathrm{p}=0.008)$. Improvements were not related to medication use; only $13 \%$ of adults were receiving medication for tics compared to $81 \%$ during childhood. In spite of substantial childhood educational or social problems, most adults were either employed or attending school full-time. One quarter of the sample was disabled as adults, with alcohol abuse, unemployment, criminal activity or other social dysfunction. Early life dysfunction closely correlated with adult dysfunction. Tics in GTS will improve over time but often persist in to adulthood. Early and assertive intervention in childhood is recommended and may serve to prevent dysfunction in adulthood. (Pappert EJ, Goetz CG, Louis ED et al. Objective assessments of longitudinal outcome in Gilles de la Tourette's syndrome. Neurology October (1 of 2) 2003;61:936-940). (Reprinis: Dr Eric J Pappert MD, 2379 NE Loop 410́, Suite 12, San Antonio, TX 78217).

COMMENT. Tourette syndrome (TS) is a relatively common neurobehavioral disorder of childhood with a prevalence of $0.7 \%$ to $4.2 \%$. TS may persist in to adulthood, but its frequency and severity are usually diminished. TS is characterized by motor and vocal tics, and is frequently associated with attention deficit/hyperactivity disorder and obsessive-compulsive disorder. Autosomal dominant, recessive, and bilineal transmission has been postulated. The co-occurrence of patients with TS and Rett syndrome in the same families has been described, suggesting a common underlying genetic defect. Screening for mutations in the MECP2 (Rett syndrome) gene in 31 male TS patients with no family history of TS found none. MECP2 gene is neither a frequent cause nor a modifier of the clinical phenotype in TS. (Rosa AL et al. Arch Neurol 2003;60:502-503). 